\title{
COMPARISON OF MINIMALLY INVASIVE SURGERY VERSUS LAPAROTOMY FOR SURGICAL STAGING OF ENDOMETRIAL CARCINOMA: A RETROSPECTIVE STUDY
}

\author{
Priyadarshini $M^{1^{*}}$, Bhat $R A^{2}$
}

\begin{abstract}
Affiliation
1. Consultant, Department of Obstetrics and Gynaecology, Birat Medical College and Teaching Hospital, Biratnagar, Nepal

2. Senior Consultant \& Head of Department, Department of Gynaecological Oncology, Health Care Global Enterprises LTD, Tower 3, P. Kalinga Rao Road, Sampangiramanagar, Bangalore, Karnataka, India
\end{abstract}

\section{ARTICLE INFO}

Received : 21 October, 2019
Accepted : 17 January, 2020
Published : 30 June, 2020

(C) Authors retain copyright and grant the journal right of first publication with the work simultaneously licensed under Creative Commons Attribution License CC - BY 4.0 that allows others to share the work with an acknowledgment of the work's authorship and initial publication in this journal.

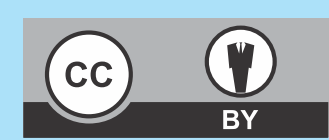

ORA 156

DOI: https://doi.org/10.3126/bjhs.v5i1.29613

\section{* Corresponding Author}

Dr. Mona Priyadarshini

DNB (Obstetrics and Gynaecology), Fellowship Gynaecology Oncology Consultant, Department of Obstetrics and Gynaecology Birat Medical College and Teaching Hospital Biratnagar, Nepal

Email: shilpy62@gmail.com

ORCID ID: https://orcid.org/0000-0003-1916-7225

\section{Citation}

Priyadarshini M, Bhat RA. Comparison of Minimally Invasive Surgery Versus Laparotomy for Surgical Staging of Endometrial Carcinoma: A Retrospective Study. BJHS 2019;5(1)11:916-920.

\section{ABSTRACT}

\section{Introduction}

Endometrial carcinoma is one of the commonest gynaecological cancer in developed countries as well as developing countries.The mainstay of initial treatment of endometrial carcinoma is surgical staging which may be performed by either the conventional abdominal approach or by minimally invasive route i.e. laparoscopic or robotic.

\section{Objectives}

The purpose of this study was to compare and evaluate the surgical staging, safety and clinical benefits of minimally invasive surgeries versus. laparotomy in patients with endometrial cancer

\section{Methodology}

We retrospectively analyzed 105 patients with endometrial cancer over a period of five years and compared the outcome of total hysterectomy with pelvic and para-aortic lymphadenectomy by abdominal, laparoscopic approach or robotic-assisted surgery. Comparison was done with respect to operative time, blood loss, number of lymph nodes retrieved, length of hospital stay, intraoperative and postoperative complications. The data were analyzed using paired " $\mathrm{t}$ " - test / Wilcoxon signed rank test , $\chi^{2}$ - test, Pearson correlation coefficient " $r$ " whenever found suitable. $p$ value of less than 0.05 was considered as statistically significant.

\section{Result}

There was no statistically significant difference seen in the baseline characteristics like age and BMI between the two groups. The laparotomies were done in a shorter time than the minimally invasive approach $(p<0.001)$. The amount of blood loss $(p=0.002)$, and the duration of hospital stay $(p<0.001)$ was significantly less in the minimally invasive surgery group than the laparotomies. Not much difference in the lymph node retrieval was observed between the two arms $(p=0.614)$. The number of complications were almost similar in both the groups.

\section{Conclusion}

Minimally invasive surgery for surgical staging of endometrial carcinoma is feasible and effective than laparotomy. The amount of blood loss and duration of hospital stay is seen much lesser with MIS than laparotomy.

\section{KEYWORDS}

Endometrial cancer; minimally invasive surgery; total abdominal hysterectomy; lymphadenectomy, laparoscopy; robotic assisted hysterectomy 


\section{INTRODUCTION}

Endometrial carcinoma is one of the commonest gynaecological cancer in developed countries while third most common gynaecological cancer in India after cervical and ovarian cancer. The GLOBOCAN 2018 had anticipated 382069 (2.1\%) new cases and 89929 (0.9\%) deaths worldwide due to endometrial cancer, while in India, 13328 (1.2\%) new cases and $5010(0.7 \%)$ deaths were estimated. ${ }^{1}$ Moreover higher incidence of the disease has been found in obese women. ${ }^{2}$ The cancer is surgically staged according to the Federation of Gynaecology and Obstetrics (FIGO) staging system. The mainstay of initial treatment is surgical management comprising of hysterectomy, bilateral salpingo-oophorectomy, pelvic and para- aortic lymph node dissection, omentectomy and peritoneal biopsies depending upon the histologic type. Planning of post operative adjuvant therapy in the form of radiotherapy and / or chemotherapy is guided by the risk stratifications. ${ }^{3}$ The surgical procedure may be performed by either the conventional abdominal approach or by minimally invasive route i.e. laparoscopic or robotic. Over the past decades, the practice has trended towards minimally invasive approach from total abdominal hysterectomy (TAH) even for surgical staging of endometrial cancers. ${ }^{2}$ Even though TAH is the conventional approach, it causes a larger abdominal incision leaving behind a visible scar which is cosmetically unappealing. Association of comorbidities like diabetes and obesity renders the wound prone to infection and increases the healing time and complications like wound dehiscence, incisional hernia etc. In 1993, the use of laparoscopy in the management of patients with endometrial cancer was first described by Childers et al. He reported two cases of laparoscopic-assisted vaginal hysterectomy (LAVH) for the management of endometrial carcinoma, since then, there has been multiple studies on the use of minimally invasive approach to perform endometrial cancer staging. ${ }^{3}$ There are many potential benefits of minimally invasive surgeries and many studies have shown decreased post operative morbidity, pain, recovery time, complications as well as an increase in patient satisfaction and quality of life associated with laparoscopic approach. ${ }^{4}$

However, the most advanced approach is the da Vinci surgical system i.e. robotic surgery, which offers certain advantage over the total laparoscopic hysterectomy (TLH). The advantage includes improved visualisation due to 3-dimensional imaging of the operating field, stability of camera with better ergonomics for operating surgeon, there is elimination of the fulcrum effect of the laparoscopy as the robotic arms imitates seven degrees of movements and eliminates hand tremors. It offers added advantage of multi tasking end wrist instrumentation. $^{5}$

The purpose of this study was to assess whether minimally invasive surgery is safer, efficacious and effective than traditional laparotomy for treatment of endometrial cancer in Indian scenario. Therefore, the present study was aimed to compare the peri-operative and oncological outcome of minimally invasive surgeries with conventional laparotomy for endometrial cancer patients.

\section{METHODOLOGY}

This retrospective observational study was conducted in the department of Gynecological Oncology at Health Care Global Enterprises, LTD, Bengaluru, India from March 2013 till March 2018. All the patients with histologically proven diagnosis of carcinoma endometrium, who underwent staging surgery during this duration were included in the study, while those with other malignancies, uterine size more than 10 weeks of gestation were excluded from the study.

The study population consisted total of 105 patients, where the open arm (laparotomy) comprised of 43 patients while 62 patients underwent minimally invasive surgery (MIS) surgery, out of which 31 underwent laparoscopy and 31 underwent robotic assisted surgery. A detailed history and examination findings were collected from the case records and a proper pro-forma was maintained which included the demographic profile of the patient depicting the unique health identification numbers (UHID number), age, Body Mass Index, date of surgery, procedure details ie. laparotomy (Total abdominal hysterectomy with bilateral salpingo- oophorectomy with +/- bilateral pelvic +/- para aortic lymphadenectomy) / laparoscopy assisted hysterectomy with bilateral salpingo- oophorectomy with +/- bilateral pelvic +/- para aortic lymphadenectomy / robotic assisted hysterectomy with bilateral salpingo- oophorectomy with +/- bilateral pelvic +/- para aortic lymphadenectomy ), stage, histology and grade of the disease, duration of surgery, estimated blood loss, number of lymph nodes obtained, number of days of hospital stay, intra operative complications and post operative complications.

Statistics - Data Analysis Plan

The data was processed through computer with statistical software SPSS 21.0 version. The continuous variables were expressed as mean and standard deviation and the categorical variables were expressed as frequency and percentage. For calculation of inferential statistics, paired " $\mathrm{t}$ "- test /Wilcoxon signed rank test , $\chi 2$ - test, Pearson correlation coefficient " $r$ " were used whenever found suitable and necessary and interpretations were made. $p$ value of less than 0.05 was considered as statistically significant.

\section{RESULTS}

Over a five years span, a total of one hundred five patients were included in the study, where forty-three underwent laparotomy whereas sixty-two underwent minimally invasive surgery for staging of endometrial cancer. A CONSORT diagram of participant flow is shown in (Figure 1). 


\section{Enrollment}

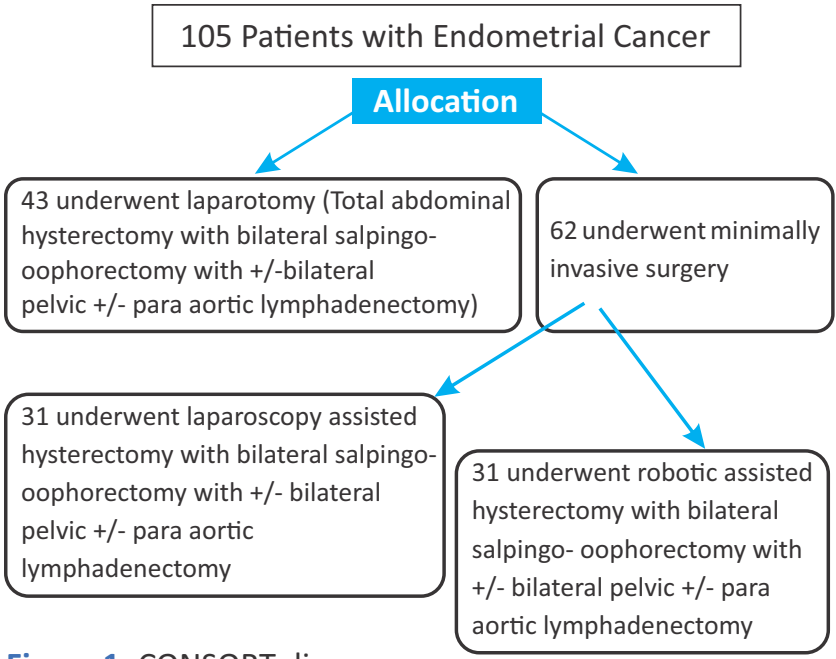

Figure 1: CONSORT diagram

Table 1 shows the comparison of age, BMI, stage and grade of the tumor between the two groups. The age of patients participating in the study ranged between 21 to 78 years. There was no significant difference observed in the mean age of the patients undergoing laparotomy and MIS (60.6 versus $57.8, p=0.134)$. Even while comparing the $B M I$, the result was insignificant (31.2 versus 30.9, $p=0.951$ ). Most of the patients in our study had early stage disease, $65.1 \%$ in the laparotomy group and $83.9 \%$ in the MIS group ( $p=0.008)$.

Table 1: Comparison between laparotomy and MIS-
Demographic profile \& pathological findings
\begin{tabular}{|c|c|c|c|}
\hline Parameters & $\begin{array}{c}\text { Laparotomy } \\
\text { (N= 43) }\end{array}$ & MIS (N=62) & p- value \\
\hline $\begin{array}{c}\text { Age (in years) } \\
\text { mean (SD) }\end{array}$ & $60.67(10.39)$ & $57.80(11.13)$ & 0.134 \\
\hline BMI mean (SD) & $31.23(5.97)$ & $30.93(6.57)$ & 0.951 \\
\hline Stage I; N(\%) & $28(65.1 \%)$ & $52(83.9 \%)$ & 0.008 \\
\hline II & $6(14 \%)$ & $0(0 \%)$ & \\
\hline III & $6(14 \%)$ & $9(14.5 \%)$ & \\
\hline IV & $3(7 \%)$ & $1(1.6 \%)$ & \\
\hline Grade ; N(\%) & & & \\
\hline I & $13(30.2 \%)$ & $34(54.8 \%)$ & 0.039 \\
\hline II & $12(27.9 \%)$ & $13(21 \%)$ & \\
\hline III & $18(41.9 \%)$ & $18(41.9 \%)$ & \\
\hline
\end{tabular}

The time duration of surgery was significantly higher $(p<0.001)$ in the MIS arm (305.5 minutes) while 220.6 minutes in laparotomy arm as depicted in Table 2 . The blood loss was considerably less ( $231.4 \mathrm{ml}$ ) in MIS than $384.1 \mathrm{ml}$ in the laparotomy arm $(p=0.002)$. Number of lymph nodes retrieved by abdominal and MIS routes showed similar result ( 25.0 versus $26.3, p=0.614)$. Patients who underwent MIS stayed for fewer days (3.81 days) in the hospital than those who opted laparotomy (5.72 days), $\mathrm{p}<0.001$ (Table 2).
Table 2: Comparison between laparotomy and MIS Perioperative outcome

\begin{tabular}{|c|c|c|c|}
\hline Parameters & $\begin{array}{c}\text { Laparotomy } \\
\text { ( N=43) }\end{array}$ & $\begin{array}{c}\text { Minimally } \\
\text { invasive surgery } \\
\text { (N=62) }\end{array}$ & $\mathrm{p}$ value \\
\hline $\begin{array}{c}\text { Surgery duration in } \\
\text { minutes; Mean(SD) }\end{array}$ & $220.69(106.49)$ & $305.58(83.07)$ & $<0.001$ \\
\hline $\begin{array}{c}\text { Blood loss in ml; } \\
\text { Mean(SD) }\end{array}$ & $384.18(164.79)$ & $231.45(197.94)$ & $\mathbf{0 . 0 0 2}$ \\
\hline $\begin{array}{c}\text { Lymph nodes; Mean } \\
\text { (SD) }\end{array}$ & $25.09(12.438)$ & $26.31(11.845)$ & $\begin{array}{c}\mathrm{p}= \\
0.614\end{array}$ \\
\hline $\begin{array}{c}\text { Hospital stay in days; } \\
\text { Mean (SD) }\end{array}$ & $5.72(2.58)$ & $3.81(1.304)$ & $<0.001$ \\
\hline
\end{tabular}

31 out of 43 laparotomies and 36 out of 62 patients who underwent MIS developed complications (Table 3). Minor complications like paralytic ileus were seen mostly in laparotomies (32.5\%) while $11.2 \%$ of the MIS developed ileus. ( $p=0.007)$. Wound gapping was the other complication which was encountered in the laparotomy group more than MIS, $25.5 \%$ versus $1.6 \%$ ( $p<0.001)$. Major complications like ureteral injury(1), bowel (1) and bladder(1) incontinence were seen in the MIS group, but were not statistically significant. Two patients developed deep vein thrombosis in each arms $(p=0.708)$. Two patients, one in each arms succumbed due to cardiopulmonary event. There were total six conversion to laparotomy, two in the robotic arm and four in laparoscopic arm. Robotic arm conversions to laparotomy were due to excessive bleeding and anaesthesia complication, while out of the four laparoscopic conversions, two were due to dense adhesions, and two due to higher BMI. Four out of forty three laparotomies and eleven out of sixty two patients who underwent minimally invasive surgery, were kept on elective ventilation.

\begin{tabular}{cccc}
\hline \multicolumn{2}{c}{ Table 3: Complications } & & \\
Parameter; N (\%) & $\begin{array}{c}\text { Laparotomy } \\
(\mathrm{N}=43)\end{array}$ & MIS (N=62) & p value \\
Overall complications & $31(72.0 \%)$ & $36(58.0 \%)$ & 0.141 \\
Ileus & $14(32.5 \%)$ & $7(11.2 \%)$ & 0.007 \\
Blood transfusion & $5(11.6 \%)$ & $7(11.2 \%)$ & 0.957 \\
Lymphedema & $3(6.9 \%)$ & $2(3.2 \%)$ & 0.375 \\
Wound Gapping & $11(25.5 \%)$ & $1(1.6 \%)$ & $<0.001$ \\
Deep vein thrombosis & $2(4.6 \%)$ & $2(3.2 \%)$ & 0.708 \\
UTI & $4(9.3 \%)$ & $3(4.8 \%)$ & 0.367 \\
Secondary resuturing & $4(9.3 \%)$ & $1(1.6 \%)$ & 0.069 \\
Hematuria & $0(0 \%)$ & $1(1.6 \%)$ & 0.403 \\
Bowel incontinence & $0(0 \%)$ & $1(1.6 \%)$ & 0.403 \\
Bladder incontinence & $0(0 \%)$ & $1(1.6 \%)$ & 0.403 \\
Vesicle vaginal fistula & $0(0 \%)$ & $1(1.6 \%)$ & 0.403 \\
Abdominal edema & $0(0 \%)$ & $1(1.6 \%)$ & 0.403 \\
Lymphocyst & $4(9.3 \%)$ & $4(6.4 \%)$ & 0.588 \\
Fever & $1(2.3 \%)$ & $0(0 \%)$ & 0.228 \\
Chest complications & $1(2.3 \%)$ & $0(0 \%)$ & 0.228 \\
Stroke & $1(2.3 \%)$ & $0(0 \%)$ & 0.228 \\
Incisional hernia & $3(6.9 \%)$ & $0(0 \%)$ & 0.035 \\
Atrial fibrillation & $1(2.3 \%)$ & $0(0 \%)$ & 0.228 \\
Vulval edema & $0(0 \%)$ & $2(3.2 \%)$ & 0.234 \\
Ureteral injury & $0(0 \%)$ & $1(1.6 \%)$ & 0.403 \\
Cardio pulmonary event & $1(2.3 \%)$ & $1(1.6 \%)$ & 0.793 \\
& & & \\
\hline & & & \\
\hline & & & \\
\hline
\end{tabular}

\section{DISCUSSION}

The purpose of any treatment offered for cancer management is to ensure least morbidity and mortality. The GOG Lap 2 study, ${ }^{4}$ which has been the largest randomised study till date, compared laparoscopy to laparotomy, and 
concluded that laparoscopic surgery is feasible and safe for treatment of endometrial cancer. In the present study, we compared and analysed the benefits of minimally invasive surgery over laparotomy.

In the present study, we did not observe any significant difference with regards to the baseline characteristics like age and BMI as also seen in many other retrospective studies. ${ }^{3,6}$ Elderly age and obesity did not pose to be a contraindication for this approach. Even though we did not observe any considerable difference in the BMI between the two groups, we successfully operated on a patient with BMI of 55.6 by robotic technique. Due to smaller wounds, lesser pain score and early ambulation, we believe that MIS can be a better choice for the obese and those who are at risk of developing venous thromboembolism. We observed that majority of the patients were in the early stage of disease, mainly stage I, and were operated by MIS approach, illustrating that the complete excision of the tumor can be feasible maintaining the surgical oncological principles.

Manchana $\mathrm{T}$ et $\mathrm{al}^{7}$ compared the perioperative and oncological outcome with laparotomy and laparoscopic or robotic surgery for women with endometrial cancer and observed that MIS has more favourable outcome in terms of blood loss, shorter hospital stay and faster recovery time than laparotomy. The operative time for laparotomy was significantly less than MIS, similar results was observed in our study, where the laparotomy was performed in a significantly lesser time than the minimally invasive procedure $(p<0.001)$. In the MIS arm, the robotic surgery had the longest operative time, which included the additional robot docking duration. We assume that if we eliminate the additional robot docking time, the actual operative time by robot would be almost equal if not less than the laparoscopy arm.

Terao $\mathrm{Y}$ et $\mathrm{al}^{8}$ studied the surgical and oncological outcome of laparoscopic surgery, compared to laparotomy for Japanese patients with endometrial cancer and concluded that the amount of blood loss and duration of hospital stay was considerably less in those operated by minimally invasive procedure than laparotomy. The observation was in line with our findings, where we found that the amount of blood loss $(p=0.002)$ was significantly less along with shorter hospital stay $(p<0.001)$ in the minimally invasive arm. The improved visualization, precision and accuracy offered by MIS techniques prompts lesser blood loss. Lymphadenectomy forms an important component of surgical staging either by laparotomy or MIS for endometrial cancer. Studies have shown different results comparing the two approaches. Some studies have reported no difference in the number of lymph nodes retrieved ${ }^{9,10}$ while some indicate the importance of MIS in obtaining increased number of lymph nodes. ${ }^{11,12}$ We observed not much difference in the lymph node yield while comparing the two approaches. At the end of our study, we observed that the overall complication rate with laparotomy was $72 \%$, while with minimally invasive surgeries was $58 \%$. Minor complications were commonly seen in the laparotomy group while there were no significant differences observed in the major complications in between the two groups.
Similar results were observed in the study conducted by Ansar P P et al. ${ }^{13}$

Prior to the decision of route of surgery, input from anaesthetist and physician is a necessity as insufflation of abdomen during MIS procedure and the intra operative position may worsen the restrictive lung function, if there is prior chronic lung disease. In our study, 11 out of 62 patients in the MIS arm and 4 out of 43 in the open surgery group were empirically put on elective ventilation by the anaesthetist in view of anticipated cerebral and laryngeal edema due to intra operative position.

The preconceived notion of uterine manipulator causing the spread of malignant cells while manipulating the uterus during laparoscopic surgery was defended by a randomized study by Lee $\mathrm{M}$ et $\mathrm{al}^{14}$ where the authors showed that there was no increase in the rate of positive peritoneal cytology or lymphovascular space invasion.

Many studies ${ }^{12,13,15}$ have affirmed that minimally invasive surgery when compared with laparotomy, results in better outcome with respect to blood loss and duration of hospital stay. However, not much difference has been appreciated in our study in terms of peri-operative complications and the oncological outcomes between the two methods. MIS is safe, feasible and effective procedure and can even be performed for the surgical staging of endometrial cancer.

\section{CONCLUSION}

Very few studies have been conducted in India to show the advantage of minimally invasive surgeries over laparotomy for the management of endometrial carcinoma. We conclude that minimally invasive surgery is as feasible as laparotomy for the surgical staging of endometrial cancer maintaining all the oncological principles and has added advantages of reduced blood loss, decreased intra and post operative complications, fewer days of hospital stay. However, we could not ascertain the advantage of robotic approach over laparoscopy surgery, except for the intra operative blood loss which was less with robotic surgery.

\section{LIMITATIONS}

Limitation of our study was the small sample size and retrospective nature of our study. Further multicentric prospective studies evaluating the advantages of minimally invasive surgery in patients with endometrial cancer in a larger population in Indian settings would be needed to validate the outcome.

\section{RECOMMENDATION}

We also recommend further researches based upon long term follow up in terms of survival and cost effectiveness of minimally invasive approach over laparotomy.

\section{ACKNOWLEDGEMENT}

None

\section{CONFLICT OF INTEREST}

None 


\section{REFERENCES}

1. Ferlay J, Ervik M, Lam F, Colombet M, Mery L, Piñeros M, Znaor A, Soerjomataram I, Bray F (2018). Global Cancer Observatory: Cancer Today. Lyon, France: International Agency for Research on Cancer. http:// gco.iarc.fr/today/data/ factsheets/populations/ 356-indiafact-sheets.pdf. December, 2018.

2. Capozzi, V. A., Sozzi, G., Gambino, G., Cianciolo, A., Riccò, M., Monfardini, L., Gaiano, M., Chiantera, V., Uccella, S., Berretta, R."Laparoscopy versus laparotomy for surgical treatment of obese women with endometrial cancer: A cost-benefit comparative analysis". Molecular and Clinical Oncology 11, no. 4 (2019): 335342. https://doi.org/10.3892/mco.2019.1901

3. Van den Bosch A, Mertens H. Implementation of laparoscopic surgery for endometrial cancer: work in progress. Facts Views Vis Obgyn. 2016 Mar 28; 8(1): 23-30. PMID: 27822348

4. Walker JL, Piedmonte MR, Spirtos NM, Eisenkop SM, Schlaerth JB, Mannel RS, Spiegel G, Barakat R, Pearl ML and Sharma SK: Laparoscopy compared with lap arotomy for comprehensive surgical staging of uterine cancer: Gynecologic onco logy group study LAP2. J Clin Oncol.27:5331-5336. 2009. doi: 10.1200/JCO. 2009.22.3248

5. Zanagnolo V, Achilarre M T, Maruccio M \& Garbi A. Might roboticassisted surgery become commonplace in endometrial cancer treatment?, Expert Review of Anticancer Therapy.2018;18:6,507509.DOI: 10.1080/14737140.2018.1469981

6. Chiou H Y, Chiu L H, Chen C H, Yen Y K, Chang C W, Liu W M. Comparing robotic surgery with laparoscopy and laparotomy for endometrial cancer management: A cohort study.International Journal of Surgery. 2015;13:17-22. https://doi.org/10.1016/ j.ijsu. 2014.11.015.

7. Manchana $T$, Puangsricharoen $P$, Sirisabya N, Worasethsin $P$, Vasuratna A, Termrungruanglert $W$, et al. Comparison of Perioperative and Oncologic Outcomes with Laparotomy, and Laparoscopic or Robotic Surgery for Women with Endometrial Cancer. Asian Pacific Journal of Cancer Prevention. 2015 Aug 3;16(13): 5483-8. http://dx.doi.org/10.7314/ APJCP.2015. 16.13.5483
8. Yasuhisa Terao, Mari Kitade, Soshi Kusunoki, Kazunari Fujino, Takafumi Ujihira, Miki Kimura, Hiroshi Kaneda, Satoru Takeda . Surgical and oncological outcome of laparoscopic surgery, compared to laparotomy, for Japanese patients with endometrial cancer. The Asia-Pacific Association for Gynecologic Endoscopy and Minimally Invasive Therapy. Volume 5, Issue 2, May 2016, Pages 64-68

9. Chu L-H, Chang W-C, Sheu B-C. Comparison of laparoscopic versus conventional open method for surgical staging of endometrial carcinoma. Taiwan J Obstet Gynecol. 2016; 55:188-92. https:// doi.org/10.1016/j.tjog.2016.02.007

10. Park, D. Clinical Effectiveness And Safety Of Robot-Assisted Laparoscopic Hysterectomy Versus Traditional Laparotomy For Endometrial Cancer: A Systematic Review. Value in Health.2015 Nov;18(7): A433. DOI: https://doi.org/10.1016/j.jval.2015.09.1039

11. Barrie A, Freeman A H, Lyon L, Garcia C, Conell C, Abbott L H et al. Classification of Postoperative Complications in Robotic-assisted Compared With Laparoscopic Hysterectomy for Endometrial Cancer. J Minim Invasive Gynecol. 2016 Nov - Dec;23(7):1181-1188. doi: 10.1016/j.jmig.2016.08.832. Epub 2016Sep 9

12. Ghazali WA, Jamil SA, Sharin IA. Laparoscopic versus laparotomy: Staging surgery for endometrial cancer - Malaysia's early experience. Gynecol Minim Invasive Ther 2019;8:25-9. doi: 10.4103 /GMIT.GMIT_25_18

13. Ansar P P, Ayyappan S, \& Mahajan, V. (2018). Prospective Nonrandomized Comparative Study of Laparoscopic Versus Open Surgical Staging for Endometrial Cancer in India. Indian journal of surgical oncology, 9(2), 133-140. https://doi.org/10.1007/ s13193017-0633-6

14. Lee M, Kim YT, Kim SW, Kim S, Kim JH, Nam EJ. Effects of uterine manipulation on surgical outcomes in laparoscopic management of endometrial cancer: a prospective randomized clinical trial. Int J Gynecol Cancer. 2013;23:372-9.

15. Ruan XC, Wong WL, Yeong HQ, Lim YKT. Comparison of outcomes following laparoscopic and open hysterectomy with pelvic lymphadenectomy for early stage endometrial carcinoma. Singapore Med J. 2018;59(7):366-369. doi:10.11622/smedj.2018088 